\title{
A dynamic linear model for the estimation of time-varying origin-destination matrices from link counts
}

\author{
Anselmo Ramalho Pitombeira-Neto ${ }^{1 *}\left(\right.$ i) and Carlos Felipe Grangeiro Loureiro ${ }^{2}$ \\ ${ }^{1}$ Department of Industrial Engineering, Federal University of Ceará, Fortaleza, Brazil \\ ${ }^{2}$ Department of Transportation Engineering, Federal University of Ceará, Fortaleza, Brazil
}

\begin{abstract}
SUMMARY
We propose a dynamic linear model (DLM) for the estimation of day-to-day time-varying origindestination (OD) matrices from link counts. Mean OD flows are assumed to vary over time as a locally constant model. We take into account variability in OD flows, route flows, and link volumes. Given a time series of observed link volumes, sequential Bayesian inference is applied in order to estimate mean OD flows. The conditions under which mean OD flows may be estimated are established, and computational studies on two benchmark transportation networks from the literature are carried out. In both cases, the DLM converged to the unobserved mean OD flows when given sufficient observations of traffic link volumes despite assuming uninformative prior OD matrices. We discuss limitations and extensions of the proposed DLM. Copyright $\odot 2017$ John Wiley \& Sons, Ltd.
\end{abstract}

KEY WORDS: OD matrices; dynamic linear models; transportation networks

\section{INTRODUCTION}

The estimation of the origin-destination (OD) matrix is an important step in planning and operating transportation systems. It consists in estimating the mean traffic flows from origin zones to destination zones in a geographical region [1]. The traditional way of estimating mean flows is by surveying people on their daily travel choices. The main caveats in these direct surveys is that their costs are high, and they are commonly carried out every decade [2].

Over the last years, the deployment of traffic control systems in urban transportation networks enabled the acquisition of large amounts of data on traffic volumes at low cost and at a high sampling rate. This sparked an increasing interest in indirectly estimating the OD matrix through mathematical models by using these data. The estimation problem may be generally defined as given a set of mean traffic volumes observed on some links in a transportation network, to estimate corresponding mean OD flows between zones of the geographic region under study. Most models developed so far assume that mean OD flows and mean traffic volumes do not vary over time and are thus termed static models.

An important issue in static models is the unique determination of OD flows given observed link volumes. Let $\boldsymbol{z}$ be a vector of observed link volumes and $\boldsymbol{x}$ be the unknown vector of corresponding OD flows (the OD matrix stretched out as a vector). As traffic volumes are sums of OD flows from several OD pairs, we must have

$$
z=\mathbf{F} \boldsymbol{x}
$$

in which $\mathbf{F}$ is an assignment matrix, whose entries give fractions of OD flows, which traverse each link. In most practical cases, the number of links in a transportation network for which traffic data is

*Correspondence to: Anselmo Ramalho Pitombeira-Neto, Departamento de Engenharia de Produção, Campus do Pici, Universidade Federal do Ceará, Fortaleza, Brasil. E-mail: anselmo.pitombeira@ufc.br 
available is smaller than the number of OD pairs, in order that the solution of the linear Equation (1) will not be unique. In other words, there may be many OD matrices consistent with observed traffic volumes.

This issue has been referred to in the literature as the underspecification of OD flows. It has been treated by some kind of regularization to the problem by using optimization models in order to uniquely determine an OD matrix. For example, Van Zuylen and Willumsen [3] and Willumsen [4] proposed non-linear programming models in which the constraints are the linear conservation flow equations given by Equation (1), and the objective function is the maximization of an entropy measure. The solution of their models provides a maximum entropy (maxent) OD matrix among those which satisfy the flow conservation equations. These maxent models are widely used in commercial packages.

Cascetta [5] proposed a generalized least squares model whose solution gives an OD matrix, which minimizes the Mahalanobis distance to a prior OD matrix known from previous studies. In order to cope with errors in observed traffic volumes, the model includes a second term in the objective function, which corresponds to the Mahalanobis distance between predicted volumes and observed volumes. Cascetta and Nguyen [6], Brenninger-Göthe and Jörnsten [7], Yang et al. [8], and Yang [9] proposed general frameworks, which include maxent and generalized least squares models as particular cases. Most optimization-related models are in some way based on these frameworks. For further developments, we refer to the papers from [10-13].

A common feature of the aforementioned models is that they do not take into account variability in OD flows. Pursuing this objective, Vardi [14] assumed OD flows are (static) random variables following independent Poisson probability distributions. He considered the fixed routing case, that is, when there is only one route for each OD pair. Given a sample of independent and identically distributed (i.i.d.) link count vectors observed in different time periods, he proposed maximum likelihood and moment-based estimators for the OD matrix. Tebaldi and West [15] proposed a Bayesian approach and used Markov chain Monte Carlo methods to estimate Poisson mean OD flows. Hazelton [16-18] extended Vardi's work by allowing multiple routes and relaxed the Poisson assumption by proposing a multivariate normal approximation to the likelihood function with an overdispersion factor. Both Bayesian and frequentist estimators were considered in his work.

In this context, the underspecification of OD flows is related to parameter identifiability in statistical models. A parameter of a model is said to be identifiable if its value could be uniquely determined from an infinite sample of observations [19]. Vardi [14] established identifiability of mean OD flows under the assumptions of independent Poisson flows, fixed routing, and that the assignment matrix has distinct nonzero columns. Manski [20] generalized this result by relaxing the Poisson and fixed routing assumptions, only requiring that OD route flows are independent, non-negative, and integer.

More recently, there has been increasing interest in dynamic models. These models do not assume that mean OD flows are static. The first efforts in this direction were concerned with what can be called within-day dynamics, whose objective is the short-term estimation of OD matrices during the course of a day. Some works in this line includes the ones by [21-23] and [24]. In these papers, the authors assume mean OD flows are non-identifiable and try to estimate deviations from known historical mean flows. Assignment matrices for all time slices in which a day is subdivided must be known or estimated. Working on a similar problem in computer networks, Singhal and Michailidis [25] have established identifiability of second-order moments under the assumption that OD flows follow multivariate normal distributions. However, they state that mean OD flows are non-identifiable.

Another line of research considers time-varying OD matrices over a sequence of days, which is referred to as day-to-day dynamics. In contrast to the within-day estimation problem, in day-to-day estimation, we aim at estimating OD matrices for a reference time period of the day, for example, the morning peak, by using past observations over many days. We assume that this reference time period is long enough so that most trips in the study region start and finish within the observation period. Nowadays, many cities around the world have traffic control systems, which acquire huge amounts of data on traffic volumes on many links in a transportation network. This allows us to have a time series of observed traffic volumes for a reference period over many days.

Hazelton [26] developed models for the estimation of day-to-day OD matrices by assuming that mean OD flows are functions of parameters that do not change over time. Particular cases include 
constant demand, linear trend, and weekday-weekend models. Parry and Hazelton [27] proposed a general Bayesian framework for the estimation of parameters of day-to-day dynamic traffic models. They assumed that OD flows vary according to a Markovian transition kernel and proposed a Markov chain Monte Carlo algorithm for parameter estimation.

In this paper, we take a different approach to the problem. We treat the sequence of observed traffic volumes over days as a time series. We model the stochastic day-to-day variation of OD flows as a dynamic linear model (DLM). This approach allows us to capture the time dependencies of OD flows. DLMs are Bayesian in nature and allow us to sequentially update the state of knowledge on OD flows every new observation on traffic volumes. In the formulation of the DLM, we model variability in traffic volumes originating from variability in OD flows, route flows, and volume measurement on links. We establish the conditions under which mean OD flows may be estimated. By means of computational experiments, we test whether the DLM is capable of estimating unobserved mean OD flows from a time series of observed link volumes given uninformative prior distributions on OD flows.

This paper is divided in the following sections: in Section 2, we describe the mathematical formulation of the DLM; in Section 3, some computational experiments are performed in order to illustrate the application of the DLM; finally, in Section 4, we discuss the results and propose further developments.

\section{DESCRIPTION OF THE PROPOSED DYNAMIC LINEAR MODEL}

In the following subsections, we describe the formulation of the DLM, model inference, and observability conditions.

\subsection{Model formulation}

Let $(\mathcal{N}, \mathcal{L})$ be a transportation network in which $\mathcal{N}$ is a set of nodes, $\mathcal{L}$ a set of directed links, and $\mathcal{J} \subseteq \mathcal{N} \times \mathcal{N}$ a set of OD pairs. For a sequence of consecutive time periods $t=1,2, \ldots, T$, we define $\boldsymbol{\theta}_{t}=\left(\theta_{t 1}, \theta_{t 2}, \ldots, \theta_{t n}\right)^{T}$ as the mean OD flow vector, in which $\theta_{t j}$ is the mean OD flow in OD pair $j \in \mathcal{J}$ at time $t$ and $n=\mid \mathcal{J}$. We define $z_{t}=\left(z_{t 1}, z_{t 2}, \ldots, z_{t m}\right)^{\mathrm{T}}$ as the vector of observed traffic volumes in a subset of links in a network at time $t$, in which $z_{t i}$ is the observed volume on link $i \in \mathcal{I} \subseteq \mathcal{L}$ and $m=|\mathcal{I}|$.

Unlike most previous models in the literature, which assume independent Poisson (or overdispersed) OD flows, we model OD flows as random variables following multivariate normal distributions. Thereby, we can benefit from a more flexible covariance structure. Moreover, the multivariate normal distribution is analytically convenient for Bayesian inference, because it is amenable to conjugacy. DLMs are Markovian state space models. We consider the vector of mean OD flows $\boldsymbol{\theta}_{t}$ as the system state, which we indirectly observe through the vector of traffic counts $\boldsymbol{z}_{t}$. A simple dynamic model for the time variation of the mean OD flow vector is based on the assumption that, in the short term, mean OD flows are locally constant. In other words, at time $t$ mean OD flows should be equal to the previous OD flows at time $t-1$ shifted by some stochastic variation $\omega_{t}$ because of the passing of time:

$$
\boldsymbol{\theta}_{t}=\boldsymbol{\theta}_{t-1}+\omega_{t}
$$

We assume that $\boldsymbol{\omega}_{t} \sim N\left(0, \mathbf{W}_{t}\right)$, where the letter " $\mathrm{N}$ " stands for the multivariate normal density with the appropriate dimension, and $\mathbf{W}_{t}$ is a covariance matrix also referred to as an evolution matrix. Notice that Equation (2) implies that mean OD flows $\boldsymbol{\theta}_{t}$ are random variables with probability density $p\left(\boldsymbol{\theta}_{t} \mid \boldsymbol{\theta}_{t-1}\right)=N\left(\boldsymbol{\theta}_{t-1}, \mathbf{W}_{t}\right)$.

In order to relate $\boldsymbol{\theta}_{t}$ and $\boldsymbol{z}_{t}$, we must take into account the distribution of OD flows over routes in a transportation network. Let $\boldsymbol{x}_{t}=\left(x_{t 1}, x_{t 2}, \ldots, x_{t n}\right)^{\mathrm{T}}$ be the vector of realized OD flows at time $t$. We assume $\boldsymbol{x}_{t} \mid \boldsymbol{\theta}_{t} \sim \mathrm{N}\left(\boldsymbol{\theta}_{t}, \boldsymbol{\Sigma}_{t}^{x}\right)$ where $\boldsymbol{\Sigma}_{t}^{x}$ is the covariance matrix of the realized OD flows, which can account for correlations among OD flows or simply be a diagonal matrix in case OD flows are independent. Given a realized vector $\boldsymbol{x}_{t}$, for each OD pair $j$, there is a vector of route flows $\boldsymbol{y}_{t j}=\left(y_{t j 1}, y_{t j 2}, \ldots, y_{t j n(j)}\right)$, in which $n(j)=\left|\mathcal{K}_{j}\right|$ is the size of the route set $\mathcal{K}_{j}$ of OD pair $j$. We assume that 
$\mathbf{y}_{t j} \mid x_{t j}, \boldsymbol{p}_{t j} \sim \mathrm{N}\left(x_{t j} \boldsymbol{p}_{t j}, \boldsymbol{\Sigma}_{t j}^{y}\right)$, in which $\boldsymbol{p}_{t j}=\left(p_{t j 1}, p_{t j 2}, \ldots, p_{t j n(j)}\right)^{\mathrm{T}}$ is the vector of route choice probabilities of OD pair $j$, and the covariance matrix $\boldsymbol{\Sigma}_{t j}^{y}$ has a multinomial-like covariance structure given by

$$
\boldsymbol{\Sigma}_{t j}^{y}=x_{t j}\left(\operatorname{diag}\left(\boldsymbol{p}_{t j}\right)-\boldsymbol{p}_{t j} \boldsymbol{p}_{t j}^{\mathrm{T}}\right)
$$

Notice also that, as $\sum_{k \in \mathcal{K}_{j}} p_{t j k}=1$, the covariance matrix $\boldsymbol{\Sigma}_{t j}^{y}$ will be singular, and the corresponding multivariate normal distribution will be degenerate. This in fact will pose no difficulty in our further development. In order to fix the degeneracy, we can specify a non-degenerate multivariate normal distribution on $n(j)-1$ components of the $\boldsymbol{y}_{t j}$ vector, for example, $\left(y_{t j 1}, y_{t j 2}, \ldots, y_{t j(n(j)-1)}\right)$, and retain only the corresponding columns and rows of the covariance matrix $\boldsymbol{\Sigma}_{t j}^{y}$, with the remaining component given by $y_{t j n(j)}=x_{t j}-\sum_{k=1}^{n(j)-1} y_{t j k}$. In large transportation networks, it may be infeasible or unnecessary to enumerate all possible routes. In this case, the covariance matrix $\boldsymbol{\Sigma}_{t j}^{y}$ includes only the terms related to a partial route set $\mathcal{K}_{j}^{\prime} \subset \mathcal{K}_{j}$. We denote as $\pi_{0 j}$ the probability of a trip occurring in a route in $\mathcal{K}_{j} \backslash \mathcal{K}_{j}^{\prime}$, so that $\sum_{k \in \mathcal{K}_{j}^{\prime}} p_{t j k}+\pi_{0 j}=1$.

Let $\boldsymbol{y}_{t}=\left(\boldsymbol{y}_{t 1}, \boldsymbol{y}_{t 2}, \ldots, \boldsymbol{y}_{t n}\right)^{\mathrm{T}}$ be the joint vector of route flows across all OD pairs. We have that $\boldsymbol{y}_{t} \mid \boldsymbol{x}_{t} \sim \mathrm{N}\left(\mathbf{P}_{t} \boldsymbol{x}_{t}, \boldsymbol{\Sigma}_{t}^{y}\right)$ because we defined multivariate normal distributions for all subvectors $\boldsymbol{y}_{t j}$ and $\mathbf{P}_{t}=$ blockdiag $_{j \in \mathcal{J}}\left\{\boldsymbol{p}_{t j}\right\}$ is a block diagonal matrix composed of the corresponding route choice probability vectors for each OD pair $j$. Because we also assume that route flow subvectors $\boldsymbol{y}_{t j}$ are conditionally independent given realized OD flow vector $\boldsymbol{x}_{t}=\left(x_{t 1}, x_{t 2}, \ldots, x_{t n}\right)^{\mathrm{T}}$ and route choice matrix $\mathbf{P}_{t}$, then $\boldsymbol{\Sigma}_{t}^{y}=\operatorname{blockdiag}_{j \in \mathcal{J}}\left\{\boldsymbol{\Sigma}_{t j}^{y}\right\}$. Now, we are able to obtain the conditional $\mathrm{p}\left(\boldsymbol{y}_{t} \boldsymbol{\theta}_{t}\right)$ by marginalizing $\boldsymbol{x}_{t}$ :

$$
\mathrm{p}\left(\boldsymbol{y}_{t} \mid \boldsymbol{\theta}_{t}\right)=\int \mathrm{p}\left(\boldsymbol{y}_{t} \mid \boldsymbol{x}_{t}\right) \mathrm{p}\left(\boldsymbol{x}_{t} \mid \boldsymbol{\theta}_{t}\right) \mathrm{d} \boldsymbol{x}_{t}
$$

Because both $\mathrm{p}\left(\boldsymbol{x}_{t} \mid \boldsymbol{\theta}_{t}\right)$ and $\mathrm{p}\left(\boldsymbol{y}_{t} \mid \boldsymbol{x}_{t}\right)$ are multivariate normal, and ignoring the dependence of $\boldsymbol{\Sigma}_{t}^{y}$ on $\boldsymbol{x}_{t}$ for now (we will take this dependence into account in the estimation equations presented in Section 2), from the properties of the multivariate normal, we have [28]:

$$
\mathrm{p}\left(\boldsymbol{y}_{t} \mid \boldsymbol{\theta}_{t}\right)=\mathrm{N}\left(\mathbf{P}_{t} \boldsymbol{\theta}_{t}, \mathbf{P}_{t} \boldsymbol{\Sigma}_{t}^{x} \mathbf{P}^{\mathrm{T}}+\boldsymbol{\Sigma}_{t}^{y}\right)
$$

In order to complete the formulation of our model, we must obtain the conditional distribution $\mathrm{p}\left(\boldsymbol{z}_{t} \mid \boldsymbol{\theta}_{t}\right)$ of observed link volumes given mean OD flows. First, we assume that $\boldsymbol{z}_{t} \mid \boldsymbol{y}_{t} \sim \mathrm{N}\left(\Delta \boldsymbol{y}_{t}, \boldsymbol{\Sigma}_{t}^{z}\right)$, where $\boldsymbol{\Sigma}_{z t}$ is the covariance matrix of the errors originated when observing volumes on links. $\Delta$ is the link-path incidence matrix for observed links, whose element $\delta_{i k}=1$ if route $k$ includes link $i$ and $\delta_{i k}=0$ otherwise. By marginalizing $\boldsymbol{y}_{t}$, we have

$$
\mathrm{p}\left(\boldsymbol{z}_{t} \mid \boldsymbol{\theta}_{t}\right)=\int \mathrm{p}\left(\boldsymbol{z}_{t} \mid \boldsymbol{y}_{t}\right) \mathrm{p}\left(\boldsymbol{y}_{t} \mid \boldsymbol{\theta}_{t}\right) \mathrm{d} \boldsymbol{y}_{t}
$$

Because both $\mathrm{p}\left(\boldsymbol{z}_{t} \mid \boldsymbol{y}_{t}\right)$ and $\mathrm{p}\left(\boldsymbol{y}_{t} \mid \boldsymbol{\theta}_{t}\right)$ are multivariate normal, we have

$$
\mathrm{p}\left(\boldsymbol{z}_{t} \mid \boldsymbol{\theta}_{t}\right)=\mathrm{N}\left(\boldsymbol{\Delta} \mathbf{P}_{t} \boldsymbol{\theta}_{t}, \boldsymbol{\Delta}\left(\mathbf{P} \boldsymbol{\Sigma}_{t}^{x} \mathbf{P}_{t}^{\mathrm{T}}+\boldsymbol{\Sigma}_{t}^{y}\right) \boldsymbol{\Delta}^{\mathrm{T}}+\boldsymbol{\Sigma}_{t}^{z}\right)
$$

Finally, calling $\mathbf{F}_{t}=\Delta \mathbf{P}_{t}$ as an assignment matrix, we can relate the mean OD flow vector $\boldsymbol{\theta}_{t}$ to the observed link volume vector $z_{t}$ as 


$$
\boldsymbol{z}_{t}=\mathbf{F}_{t} \boldsymbol{\theta}_{t}+\boldsymbol{v}_{t}
$$

where $\boldsymbol{v}_{t} \sim \mathrm{N}\left(0, \mathbf{V}_{t}\right)$ is a zero-mean error term, which corresponds to the deviation of the observed volumes relative to the expected value $\mathrm{E}\left[\boldsymbol{z}_{t} \mid \boldsymbol{\theta}_{t}\right]=\mathbf{F}_{t} \boldsymbol{\theta}_{t}$, with covariance matrix given by

$$
\mathbf{V}_{t}=\mathbf{F}_{t} \boldsymbol{\Sigma}_{t}^{x} \mathbf{F}_{t}^{\mathrm{T}}+\Delta \boldsymbol{\Sigma}_{t}^{y} \boldsymbol{\Delta}^{\mathrm{T}}+\boldsymbol{\Sigma}_{t}^{z}
$$

We draw attention to the fact that, although $\boldsymbol{\Sigma}_{t}^{y}$ may be singular, $\mathbf{V}_{t}$ will not be in most cases. Then the density $\mathrm{p}\left(\boldsymbol{z}_{t} \mid \boldsymbol{\theta}_{t}\right)=\mathrm{N}\left(\mathbf{F}_{t} \boldsymbol{\theta}_{t}, \mathbf{V}_{t}\right)$ will be non-degenerate. It is also noteworthy in Equation (5) that variability in OD flows, in route flows, and in volume measurement is represented in the covariance matrix. Together, Equations (2), (4), and (5) specify the proposed DLM. In next subsection, we develop the inference equations for mean OD flows.

\subsection{Bayesian inference on mean OD flows}

At a time $t$, we denote by $z_{0: t-1}=\left\{z_{0}, z_{1}, \ldots, z_{t-1}\right\}$ the set of observed volume vectors in past time periods and $z_{0: t}=z_{0: t-1} \cap\left\{z_{t}\right\}$ as the set of observed volume vectors including the current observed $\boldsymbol{z}_{t}$. We assume that covariance matrices $\boldsymbol{\Sigma}_{t}^{x}, \boldsymbol{\Sigma}_{t}^{z}$, and $\mathbf{W}_{t}$, the link-path incidence matrix $\boldsymbol{\Delta}$, and the route choice matrices $\mathbf{P}_{t}$ are known parameters. (In Section 4, we discuss some ways to estimate these parameters). We do inference on mean OD flows $\boldsymbol{\theta}_{t}$.

At time $t-1$, we have a posterior distribution $\mathrm{p}\left(\boldsymbol{\theta}_{t-1} \mid z_{0: t-1}\right)=\mathrm{N}\left(\boldsymbol{m}_{t-1}, \boldsymbol{C}_{t-1}\right)$, which synthesizes an analyst's knowledge on mean OD flows after observation of $z_{0: t-1}$. Then, at time $t$ we have a prior distribution $\mathrm{p}\left(\boldsymbol{\theta}_{t} \mid \boldsymbol{z}_{0: t-1}\right)=\mathrm{N}\left(\overline{\boldsymbol{m}}_{t}, \overline{\boldsymbol{C}}_{t}\right)$, which synthesizes knowledge before observation of $\boldsymbol{z}_{t}$, where $\overline{\boldsymbol{m}}_{t}=\boldsymbol{m}_{t-1}$ and $\overline{\boldsymbol{C}}_{t}=\boldsymbol{C}_{t-1}+\mathbf{W}_{t}$. Notice that uncertainty increases by the passing of time by the addition of the evolution matrix $\mathbf{W}_{t}$ to the prior covariance matrix $\boldsymbol{C}_{t-1}$.

We define the one-step forecast distribution of the vector of observed link volumes $\boldsymbol{z}_{t}$ to be $\mathrm{p}\left(\boldsymbol{z}_{t} \mid z_{0: t-1}\right)=\mathrm{N}\left(\boldsymbol{f}_{t}, \boldsymbol{Q}_{t}\right)$ where $\boldsymbol{f}_{t}=\mathbf{F}_{t} \overline{\boldsymbol{m}}_{t}$ and $\boldsymbol{Q}_{t}=\mathbf{F}_{t} \overline{\boldsymbol{C}}_{t} \mathbf{F}_{t}^{\mathrm{T}}+\mathbf{V}_{t}$. It is worth noting that in computing $\mathbf{V}_{t}$ by Equation (5), we need to compute covariance matrices $\boldsymbol{\Sigma}_{t j}^{y}$ of route flows by Equation (3). Because we do not directly observe realized OD flows $\boldsymbol{x}_{t}=\left(x_{t 1}, x_{t 2}, \ldots, x_{t n}\right)^{\mathrm{T}}$, we substitute them for the estimate $\overline{\boldsymbol{m}}_{t}$. Then we have

$$
\hat{\boldsymbol{\Sigma}}_{t j}^{y}=\bar{m}_{t j}\left(\operatorname{diag}\left(\boldsymbol{p}_{t j}\right)-\boldsymbol{p}_{t j} \boldsymbol{p}_{t j}^{\mathrm{T}}\right)
$$

for all OD pairs $j$ and $\hat{\boldsymbol{\Sigma}}_{t}^{y}=$ blockdiag $_{j \in \mathcal{J}}\left\{\hat{\boldsymbol{\Sigma}}_{t j}^{y}\right\}$. Finally, the parameters of the posterior distribution $\mathrm{p}\left(\boldsymbol{\theta}_{t} \mid \boldsymbol{z}_{0: t}\right)=\mathrm{N}\left(\boldsymbol{m}_{t}, \boldsymbol{C}_{t}\right)$ are given by [29]

$$
\begin{gathered}
\boldsymbol{m}_{t}=\boldsymbol{m}_{t}+\mathbf{A}_{t}\left(\boldsymbol{z}_{t}-\boldsymbol{f}_{t}\right) \\
\boldsymbol{C}_{t}=\overline{\boldsymbol{C}}_{t}-\mathbf{A}_{\mathrm{t}} \mathbf{Q}_{\mathrm{t}} \mathbf{A}_{t}^{\mathrm{T}}
\end{gathered}
$$

where $\mathbf{A}_{t}=\bar{C}_{t} \mathbf{F}_{t}^{\mathrm{T}} \mathbf{Q}_{t}^{-1}$ is an adjustment matrix, which controls how the parameters from the posterior distribution are modified according to the new observation $z_{t}$. In particular, the adjustment matrix is a function of the prior covariance matrix $\overline{\mathbf{C}}_{t}$ and of the inverse $\mathbf{Q}_{t}^{-1}$ of the covariance matrix of the one-step forecast distribution of the link volumes, so that the adjustment matrix gives more or less weight to the observed link volumes according to their uncertainty relative to the uncertainty in OD flows.

The inference procedure starts at time $t=0$ from a distribution $\mathrm{p}\left(\boldsymbol{\theta}_{0} \mid \boldsymbol{z}_{0}\right)=\mathrm{N}\left(\boldsymbol{m}_{0}, \mathbf{C}_{0}\right)$ where $\boldsymbol{z}_{0}$ are not actually observed link volumes, but symbolically represents the modeler's prior knowledge on OD flows. $\boldsymbol{m}_{0}$ is a prior OD matrix (or our best guess for it), before any observation of link volumes, and $\mathbf{C}_{0}$ is a covariance matrix, which synthesizes our uncertainty on the prior OD matrix $\boldsymbol{m}_{0}$. Then, for each time $t \geq 1$, we compute the parameters $\boldsymbol{m}_{t}$ and $\mathbf{C}_{t}$ of the posterior distribution according to Equations (6) and (7), where $\hat{\boldsymbol{\theta}}_{t}=\boldsymbol{m}_{t}$ is an estimator of the mean OD flows at time $t$. It is worth noting that this procedure is applied sequentially one vector of observed volumes at a time, without the need to gather a large sample of observed vectors before applying it. 


\subsection{Identifiability of mean OD flows}

Parameter identifiability is concerned with the question whether model parameters can eventually be inferred from data. As in our model mean OD flows $\boldsymbol{\theta}_{t}$ are varying in time, we cannot exactly know their values from a single observation $z_{t}$. On the other hand both $z_{t}$ and the assignment matrix $\mathbf{F}_{t}$ give information on $\boldsymbol{\theta}_{t}$, so that we expect the posterior distribution $\mathrm{p}\left(\boldsymbol{\theta}_{t} \mid \boldsymbol{z}_{t}\right)$ to be more informative than the prior $\mathrm{p}\left(\boldsymbol{\theta}_{t} \mid \boldsymbol{z}_{0: t-1}\right)$.

We do not investigate directly the increase in information from the Bayesian updating of prior to posterior. Instead, we investigate if it would be possible to know exactly the value of mean OD flows as we gather more observations over time given that they are constant, that is, $\boldsymbol{\theta}_{1}=\boldsymbol{\theta}_{2} \ldots=\boldsymbol{\theta}$, which implies that $\omega_{t}=0$ in our local constant model given by Equation (2) for $t=1,2, \ldots$. In addition, we assume that there are no observation errors, that is, $\boldsymbol{v}_{t}=0$ in Equation (4) for $t=1,2, \ldots$, in order that $\boldsymbol{z}_{t}=\mathrm{E}\left[\boldsymbol{z}_{t} \mid \boldsymbol{\theta}\right]=\mathbf{F}_{t} \boldsymbol{\theta}$. This greatly simplifies our analysis.

We notice that for a finite time horizon $t=1,2, \ldots, T$ we have

$$
\widetilde{\mathbf{z}}_{T}=\widetilde{\mathbf{F}}_{T} \boldsymbol{\theta}
$$

where $\widetilde{\mathbf{z}}_{T}=\left(\boldsymbol{z}_{1}, \boldsymbol{z}_{2}, \ldots, \boldsymbol{z}_{T}\right)^{\mathrm{T}}$ and $\widetilde{\mathbf{F}}_{T}=\left(\mathbf{F}_{1}, \mathbf{F}_{2}, \ldots, \mathbf{F}_{T}\right)^{\mathrm{T}}$. If $\operatorname{rank}\left(\widetilde{\mathbf{F}}_{T}\right)=n$, in which $n$ is the number of OD pairs, we may uniquely determine the mean OD flows by the least squares solution:

$$
\boldsymbol{\theta}=\left(\widetilde{\mathbf{F}}_{T}^{\mathrm{T}} \widetilde{\mathbf{F}}_{T}\right)^{-1} \widetilde{\mathbf{F}}_{T} \widetilde{\boldsymbol{z}}_{T}
$$

provided that $T \geq[n / m]$, since $\widetilde{\mathbf{F}}_{T}$ is a $(T m) \times n$ matrix and rank $\left(\widetilde{\mathbf{F}}_{T}\right) \leq \min (T m, n)$. A sufficient condition for $\operatorname{rank}\left(\widetilde{\mathbf{F}}_{T}\right)=n$ is that $\mathbf{F}_{1}, \mathbf{F}_{2}, \ldots, \mathbf{F}_{T}$ be all different, which is very likely in practice since transportation systems are dynamic.

With mean OD flows varying over time according to the local constant model from Equation (2), this result indicates that at time $t^{\prime} \geq t+[n / m]$ we can reasonably approximate mean OD flows $\boldsymbol{\theta}_{t}$ from Equation (8) provided they have not changed considerably over time, and this approximation is better the lower the drift in mean levels caused by $\omega_{t}$.

In next section, we illustrate by computational experiments our proposed DLM.

\section{COMPUTATIONAL EXPERIMENTS}

In the following subsections, we test the formulated DLM in two computational experiments: the first on a small network in order to illustrate some concepts; and the second on the larger network Sioux Falls, often used as benchmark in the literature. The purpose of this second experiment is to evaluate if the DLM converges to the unobserved simulated OD flows in a larger network if started from an uninformative prior OD matrix.

\subsection{A small network}

In order to illustrate the application of our proposed DLM to the estimation of day-to-day OD flows, we simulate dynamic OD flows in a small network from the paper of Hazelton [16], which is shown in Figure 1:

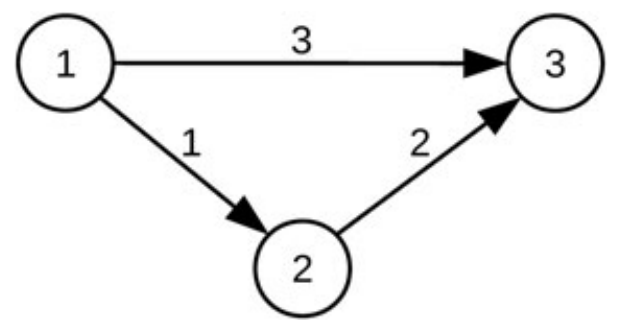

Figure 1. Network used in computational experiment 1. 
There are three OD pairs: $(1,2),(1,3)$, and $(2,3)$. OD pairs $(1,2)$ and $(2,3)$ have only one route each, and OD pair $(1,3)$ has two available routes: route 1 runs through links 1 and 2, and route 2 directly through link 3. All links have the same length of one unit. In our experiment, we assume a local constant model as given by Equation (2) and that only volumes on link 2 are observed. The assignment matrix (a row vector in this case) is given by $\mathbf{F}_{t}=\left(f_{t}^{(1,3)}, f_{t}^{(2,3)}\right)$, where $f_{t}^{(1,3)}$ and $f_{t}^{(2,3)}$ are respectively the mean fractions of OD flows from OD pairs $(1,3)$ and $(2,3)$ that pass through link 2 . We draw attention to the fact that mean OD flows for OD pair $(1,2)$ cannot be estimated from observing only volumes on link 2 because there is no route for $(1,2)$, which includes link 2 . Then mean OD flows $\boldsymbol{\theta}_{t}=\left(\theta_{t}^{(1,3)}, \theta_{t}^{(2,3)}\right)^{\mathrm{T}}$ and observed volumes $z_{t}^{(2)}$ on link 2 are given by the following DLM:

$$
\begin{gathered}
\theta_{t}^{(1,3)}=\theta_{t-1}^{(1,3)}+\omega_{t}^{(1,3)} \\
\theta_{t}^{(2,3)}=\theta_{t-1}^{(2,3)}+\omega_{t}^{(2,3)} \\
z_{t}^{(2)}=f_{t}^{(1,3)} \theta_{t}^{(1,3)}+f_{t}^{(2,3)} \theta_{t}^{(2,3)}+v_{t}^{(2)}
\end{gathered}
$$

where $\boldsymbol{\omega}_{t}=\left(\omega_{t}^{(1,3)}, \omega_{t}^{(2,3)}\right)^{\mathrm{T}} \sim \mathrm{N}\left(0, \mathbf{W}_{t}\right), v_{t}^{(2)} \sim \mathrm{N}\left(0, V_{t}\right)$, and the variance $V_{t}$ is given by Equation (5).

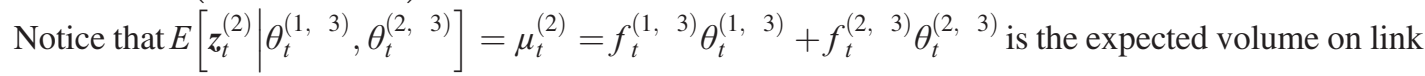
2 and $v_{t}^{(2)}$ is the observation error because of variability in OD flow generation, in route choices and in traffic measurement. Notice also that for this network $f_{t}^{(1,3)}=p_{t 1}^{(1,3)}$ and $f_{t}^{(2,3)}=p_{t 1}^{(2,3)}$, where $p_{t 1}^{(1,3)}$ and $p_{t 1}^{(2,3)}$ correspond to the probability of choosing route 1 of OD pair $(1,3)$ and route 1 of OD pair $(2,3)$ at time $t$, respectively.

In the simulation of OD flows and traffic volumes, we assume a very small drift in mean OD flows with $\mathbf{W}_{t}=\mathbf{I}$ (the identity matrix). We also assume the variances $\boldsymbol{\Sigma}_{t}^{x}=\boldsymbol{\Sigma}_{t}^{z}=\mathbf{I}$, so that the variability of actual OD flows around expected flows and variability stemming from errors in link counts are small. Most variability in actual traffic volumes on links will be due the variability in route choice through the covariance matrix $\boldsymbol{\Sigma}_{t}^{y}$. Route choice probabilities are sampled through Dirichlet distribution. We define by $\pi_{1}^{(1,3)}$ and $\pi_{2}^{(1,3)}$ mean probabilities of choosing routes 1 and 2 of OD pair $(1,3)$, respectively, which are set from a multinomial logit model where the expected utility of each route is the negative of the length of the route and scale parameter $\xi=1.0$, that is, $\pi_{1}^{(1,3)}=e^{-2} /\left(e^{-2}+e^{-1}\right)=0.2689$ and $\pi_{2}^{(1,3)}=1-\pi_{1}^{(1,3)}=0.7311$. We then have $\boldsymbol{p}_{t}^{(1,3)} \sim \operatorname{Dir}\left(\alpha_{1}^{(1,3)}, \alpha_{2}^{(1,3)}\right)$ for $t=1, \ldots, T$, with $\alpha_{1}^{(1,3)}=26.89$ and $\alpha_{2}^{(1,3)}=73.11$. The simulation starts at $\theta_{0}=(70,100,80)^{\mathrm{T}}$, and we sequentially simulate $\boldsymbol{\theta}_{t}$ and $\boldsymbol{z}_{t}$ according to Equations (9), (10), and (11).

In order to estimate mean OD flows $\boldsymbol{\theta}_{t}=\left(\theta_{t}^{(1,3)}, \theta_{t}^{(2,3)}\right)^{\mathrm{T}}$ and mean volumes $\mu_{t}^{(2)}$ on link 2, we apply Equations (6) and (7) sequentially for $t=1 \ldots T$ to determine posterior means $\boldsymbol{m}_{t}=\left(m_{t}^{(1,3)}, m_{t}^{(2,3)}\right)^{\mathrm{T}}$, which we take as estimators of mean OD flows, and posterior covariance matrices $\mathbf{C}_{t}$, which are useful to calculate credible intervals. We assume that the covariance matrices $\boldsymbol{\Sigma}_{t}^{x}$ and $\boldsymbol{\Sigma}_{t}^{z}$ and the assignment matrix $\mathbf{F}_{t}$ are known for all t. We use an evolution matrix $\mathbf{W}_{t}=10 \mathbf{I}$, which is 10 times the one used in the simulation, because in a few cases we observed non-convergence of the estimators when using $\mathbf{W}_{t}=\mathbf{I}$. The effect of using a greater evolution covariance is that the convergence process is slower. The estimator of the volumes is given by $\hat{\mu}_{t}^{(2)}=f_{t}^{(1,3)} m_{t}^{(1,3)}+f_{t}^{(2,3)} m_{t}^{(2,3)}$. Notice that the number of OD pairs for which we try to estimate mean flows is greater than the number of links we observe, a situation which is typical in practice. We start at $m_{0}=(10,10,10)$ (we retain the value for OD pair $(1,2)$ only for completion), which is far from the simulated initial 
mean values, and a diffuse covariance matrix $\mathbf{C}_{0}=10^{4} \mathbf{I}$. This means that we are quite uncertain on the actual mean OD flows.

Figure 2 shows simulated observed volumes $z_{t}^{(2)}$, simulated mean volumes $\mu_{t}^{(2)}$, and estimated mean volumes $\hat{\mu}_{t}^{(2)}$ for one simulated sample path with $T=100$. It can be seen that over the initial time steps, the estimate of link volumes given by $\hat{\mu}_{t}^{(2)}$ is far from the simulated mean volumes, because of the influence of the diffuse prior distribution on the mean OD flows. As more observations of traffic volumes $z_{t}^{(2)}$ are gathered, the estimates get closer to the simulated ones. Approximately, from $t=20 \mathrm{on}$, the estimates are reasonably close to the simulated mean volumes.

Figures 3 and 4 show the results for OD pairs $(1,3)$ and $(2,3)$, respectively. It can be seen that the posterior means $m_{t}^{(1,3)}$ and $m_{t}^{(2,3)}$ reasonably converged to the simulated mean OD flows after some time steps. Figures 3 and 4 also show the $95 \%$ credible intervals for the OD flows, which become tighter with the time steps as the posterior variances decrease over the time.

In order to evaluate the convergence across many replications, we replicated the simulation $R=100$ times for $T=10,30,100$, and 300 . We took as estimation error measure the relative absolute error at

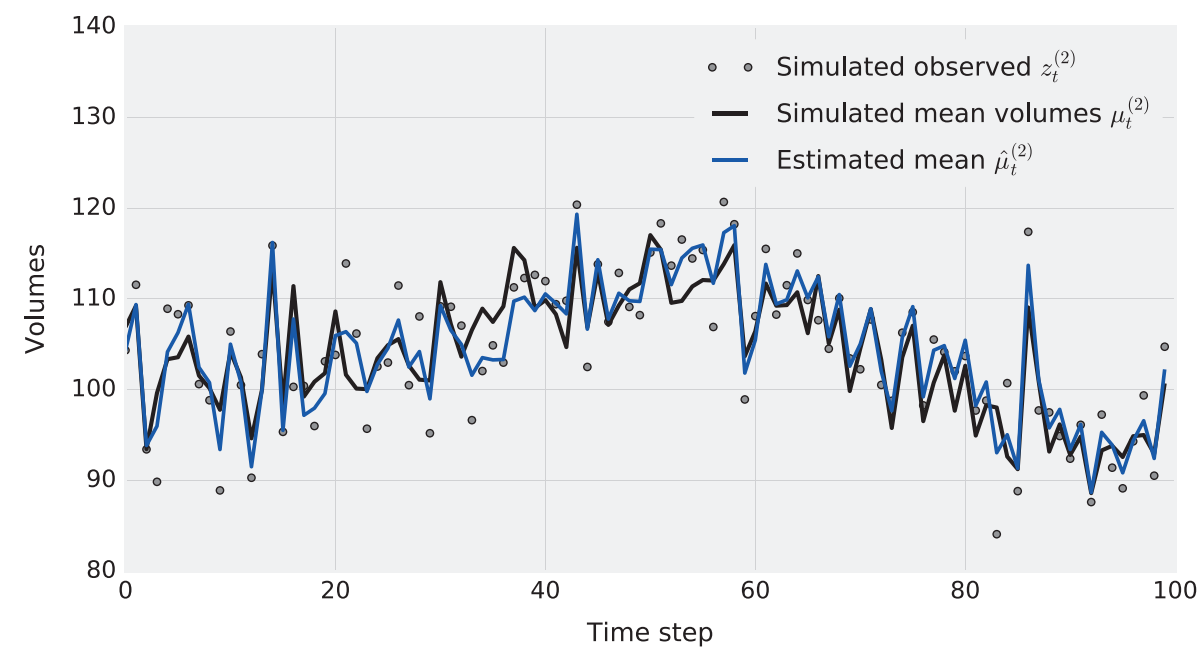

Figure 2. Simulated sample path for link 2.

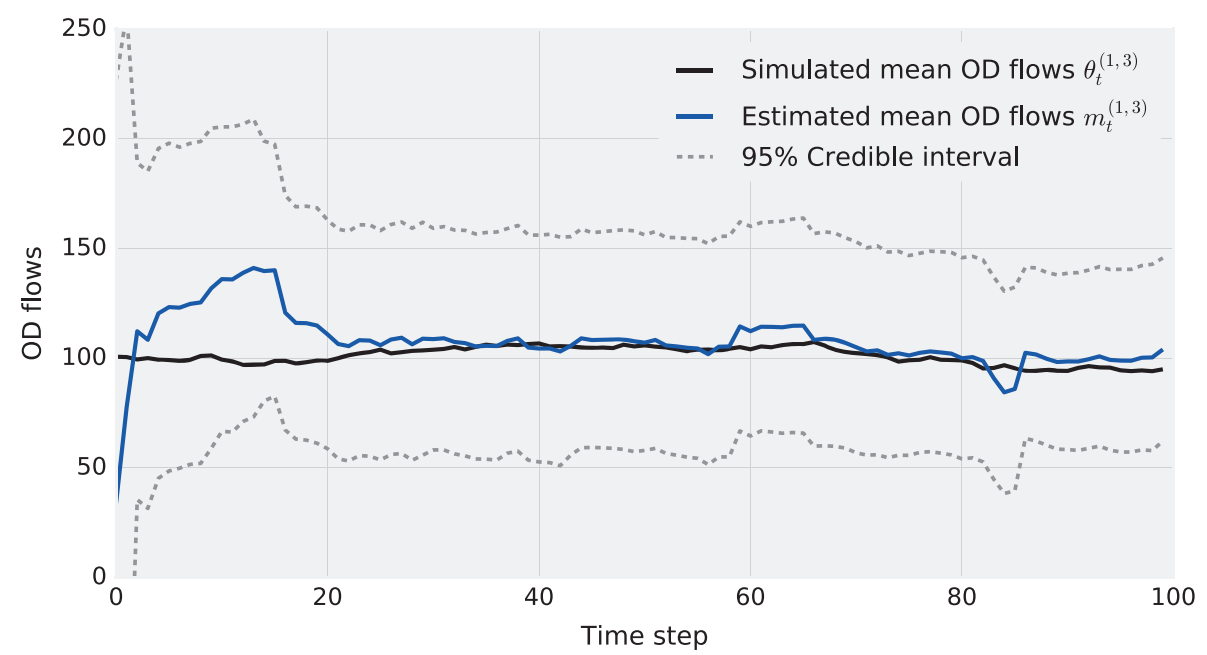

Figure 3. Simulated sample path for origin-destination (OD) pair $(1,3)$. 


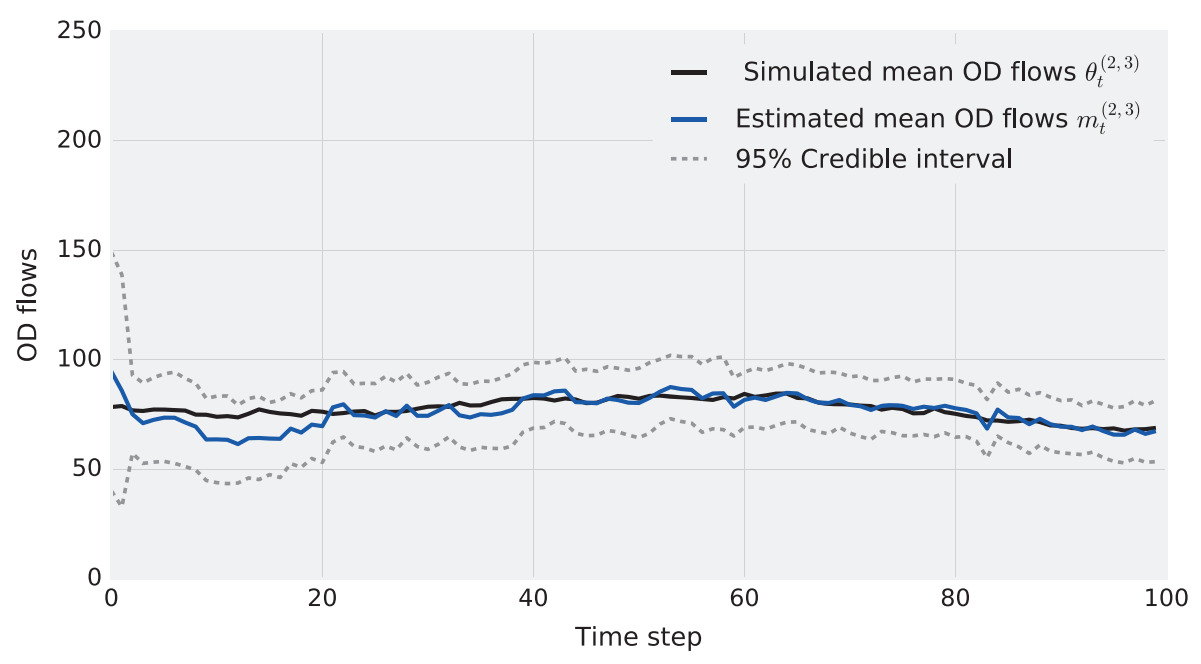

Figure 4. Simulated sample path for origin-destination (OD) pair $(2,3)$.

$t=T$ for each simulation and calculated its mean across replications, which we call the mean relative absolute error at time $T$ for OD pair $j\left(M R A E_{T}^{(j)}\right)$ :

$$
\operatorname{MRAE}_{T}^{(j)}=\frac{1}{R} \sum_{r=1}^{R} \frac{\left|m_{T}^{(j, r)}-\theta_{T}^{(j, r)}\right|}{\left|\theta_{T}^{(j, r)}\right|}
$$

We can see from Table I that the $M R A E_{T}$ becomes smaller as the simulation length $T$ increases. At $T=0$, we have the relative absolute error for the initial guess $m_{0}=(10,10,10)^{\mathrm{T}}$ in relation to the starting $\boldsymbol{\theta}_{0}=(70,100,80)^{\mathrm{T}}$. With only one observation $(T=1)$, the $M R A E_{T}$ shows a decrease to 0.6688 and 0.22909 for OD pairs $(1,3)$ and $(2,3)$, respectively, and after 100 observations, it has converged around 0.1047 and 0.0394 . These results indicate that the DLM was able to progressively converge to the unobserved mean OD flows as more traffic volumes were observed. It is interesting that the estimation error is greater for OD pair $(1,3)$, which may be due to the existence of two routes, while $(2,3)$ has only one route.

In next section, we explore the application of our DLM in the larger Sioux Falls network.

\subsection{Sioux Falls network}

In order to evaluate the performance of the DLM in a network of non-trivial size, we test its application in the well-known Sioux Falls network. Figure 5 shows a schematic representation, while Table II shows its main features.

As there are many available routes for each OD pair, we used a restricted route choice set $\mathcal{K}_{j}^{\prime}$ including only the five shortest routes for all OD pairs $j$. We define by $\pi_{k j}$ with $k=1,2, \ldots, 5$ the mean probability

Table I. $M R A E_{T}$ for the small network with $R=100$ (standard deviation in parentheses).

\begin{tabular}{lcr}
\hline & \multicolumn{3}{c}{ Origin-destination pair } \\
\cline { 2 - 3 } $\mathrm{T}$ & $(1,3)$ & $(2,3)$ \\
\hline 0 & $0.9000(0.0000)$ & $0.8750(0.0000)$ \\
1 & $0.6688(0.0404)$ & $0.2209(0.0544)$ \\
10 & $0.2703(0.2236)$ & $0.0932(0.0749)$ \\
30 & $0.1611(0.1145)$ & $0.0568(0.0453)$ \\
100 & $0.1047(0.0816)$ & $0.0394(0.0314)$ \\
300 & $0.1086(0.0806)$ & $0.0393(0.0350)$ \\
\hline
\end{tabular}




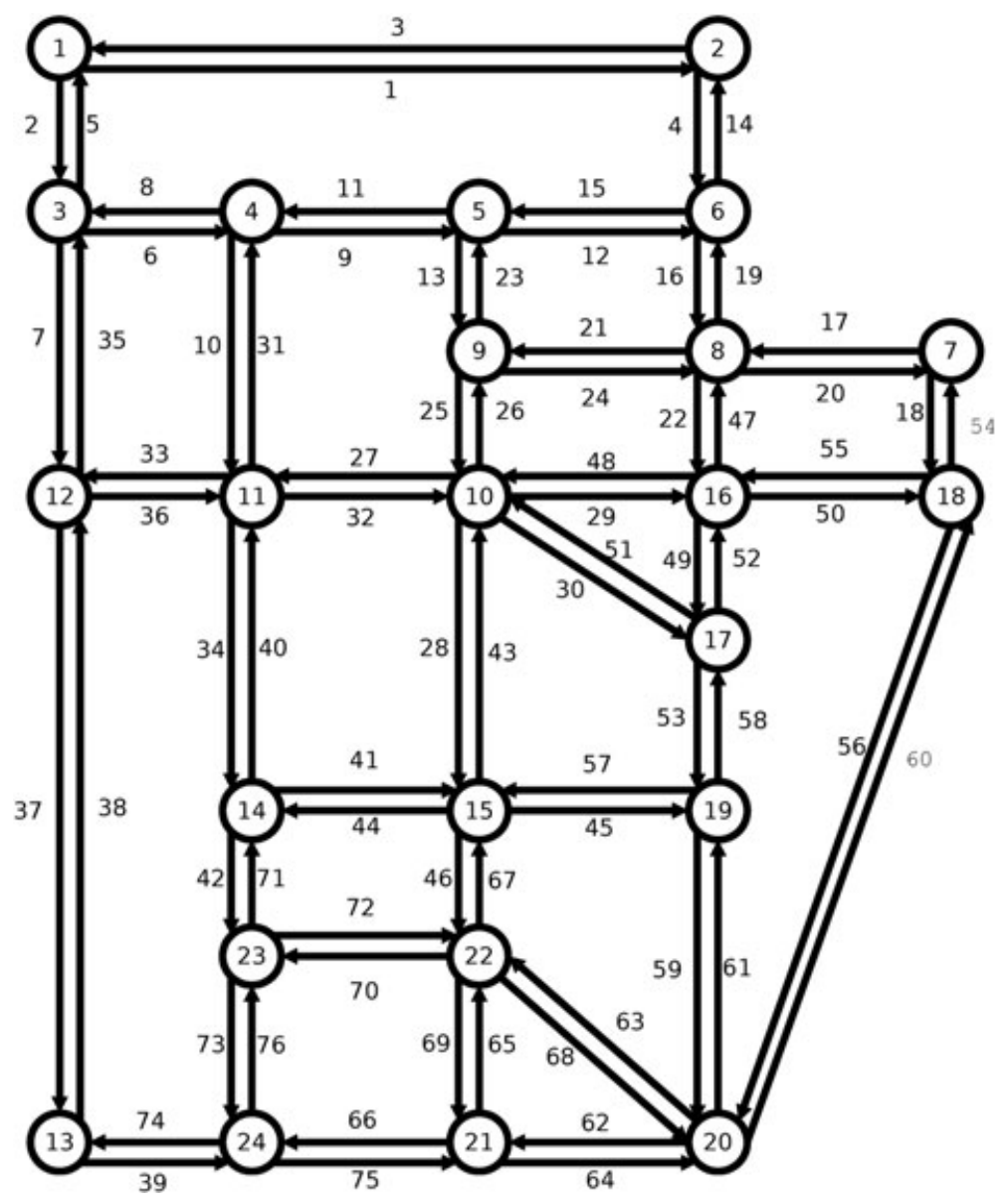

Figure 5. Schematic representation of Sioux Falls network.

Table II. Main characteristics of Sioux Falls network.

\begin{tabular}{lr}
\hline Feature & Value \\
\hline Nodes & 24 \\
Links & 76 \\
Origin-destination pairs & 552 \\
Type of link & All bidirectional \\
Total number of routes & $\approx 1.8 \times 10^{6}$ \\
\hline
\end{tabular}

of a route $k \in \mathcal{K}_{j}^{\prime}$ being chosen. We also assume a positive probability $\pi_{0 j}=0.01$ for all $j$ of a route in $\mathcal{K}_{\mathcal{J}} \mathcal{K}_{j}^{\prime}$ being selected. The mean probabilities $\pi_{k j}$ are set through a multinomial logit model where the expected utility is the negative of the length $l_{k j}$ of each route in the restricted route choice set and scale parameter $\xi=10.0$, that is, $\pi_{k j}=\left(1-\pi_{0 j}\right) e^{-l_{k j} / \xi} / \sum_{s=1}^{5} e^{-l_{s j} / \xi}$. The multiplication by $\left(1-\pi_{0 j}\right)$ is required in order to satisfy $\sum_{k=0}^{5} \pi_{k j}=1$. At each time $t$, for each OD pair $j$, we simulate the route choice probability vector according to a Dirichlet distribution, that is, $\boldsymbol{p}_{t j} \sim \operatorname{Dir}\left(\boldsymbol{\alpha}_{j}\right)$ and $\boldsymbol{\alpha}_{j}$ is a vector with components $\alpha_{k j}=\pi_{k j} / \pi_{0 j}$. The assignment matrices are set as $\mathbf{F}_{t}=\boldsymbol{\Delta} \mathbf{P}_{t}$ and $\mathbf{P}_{t}=$ blockdiag $_{j \in \mathcal{J}}\left\{\boldsymbol{p}_{t j}\right\}$. We assume an evolution matrix $\mathbf{W}_{t}=\mathbf{I}$ so as to have a very small variation in mean OD flows over time. We also adopted $\boldsymbol{\Sigma}_{t}^{x}=\boldsymbol{\Sigma}_{t}^{z}=\mathbf{I}$. Starting from $\boldsymbol{\theta}_{0}$ equals to the OD matrix available in the literature as the "real" OD matrix, for each time $t=1,2, \ldots, T$, we sample $\boldsymbol{\theta}_{t} \sim \mathrm{N}\left(\boldsymbol{\theta}_{t-1}, \mathbf{W}\right)$ and $\boldsymbol{z}_{t} \sim \mathrm{N}\left(\mathbf{F}_{t} \boldsymbol{\theta}_{t}, \mathbf{V}_{t}\right)$ with $\mathbf{V}_{t}$ given by Equation (5). 
In the estimation phase, we assume all links are observed. Although this may not be realistic, the number of OD flows to be estimated is still greater than the number of observed links, which is the typical situation in practice. We also assume that the assignment matrices $\mathbf{F}_{t}$ and covariance matrices $\boldsymbol{\Sigma}_{t}^{x}$ and $\boldsymbol{\Sigma}_{t}^{z}$ are known. We set the evolution matrix $\mathbf{W}=10.0$ (10 times greater than the one used to simulate the data), because we experienced some cases in which the estimator did not converge when we used a very small value. We start from an uninformative prior OD matrix $\boldsymbol{m}_{0}=10 \mathbf{1}$ (where $\mathbf{1}$ denotes a vector whose all components are equal to 1 ) and covariance matrix $10^{4} \mathbf{I}$, which represents the lack of knowledge of an analyst with respect to the magnitude of the OD flows. We then sequentially applied the Equations (6) and (7) after each observed vector $\boldsymbol{z}_{t}$. Figures 6 and 7 show OD flows on two sample OD pairs for $T=300$.

We repeated the simulation $R=30$ times with varying length $T$ and evaluated the mean relative absolute error at time $T$ over the replications. As we have 552 OD pairs, we considered the L1 norm of the OD flow vector, so that the $M R A E_{T}$ is defined as

$$
\operatorname{MRAE}_{T}=\frac{1}{R} \sum_{r=1}^{R} \frac{\left\|\boldsymbol{m}_{T}^{(r)}-\boldsymbol{\theta}_{T}^{(r)}\right\|_{1}}{\left\|\boldsymbol{\theta}_{T}^{(r)}\right\|_{1}}
$$

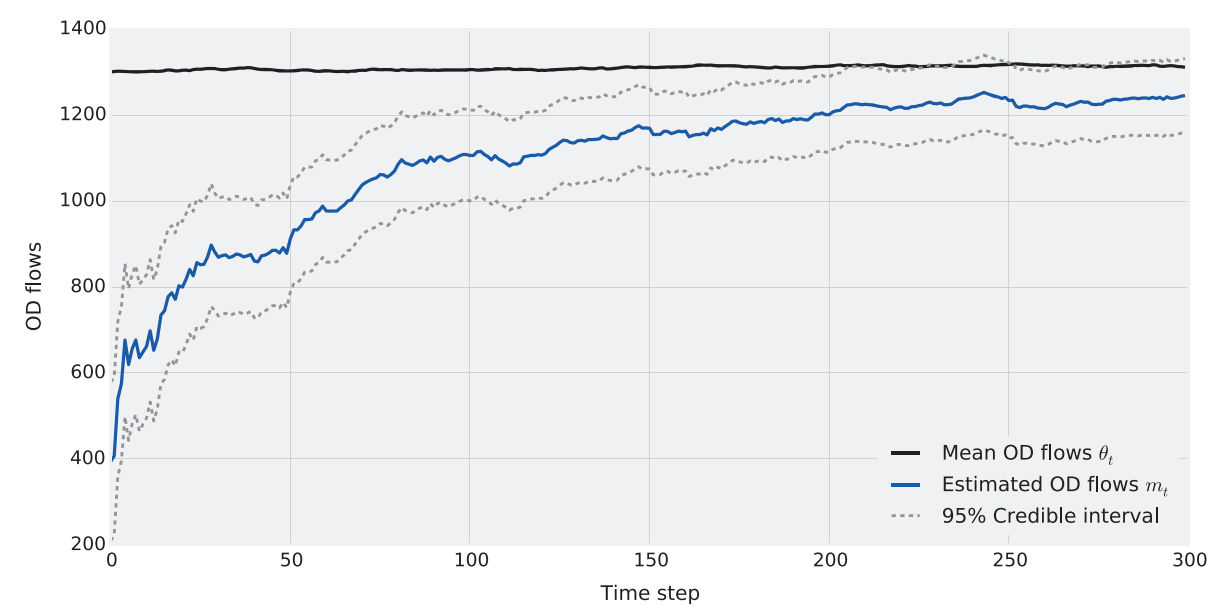

Figure 6. Origin-destination (OD) flows on OD pair $1 \rightarrow 10$.

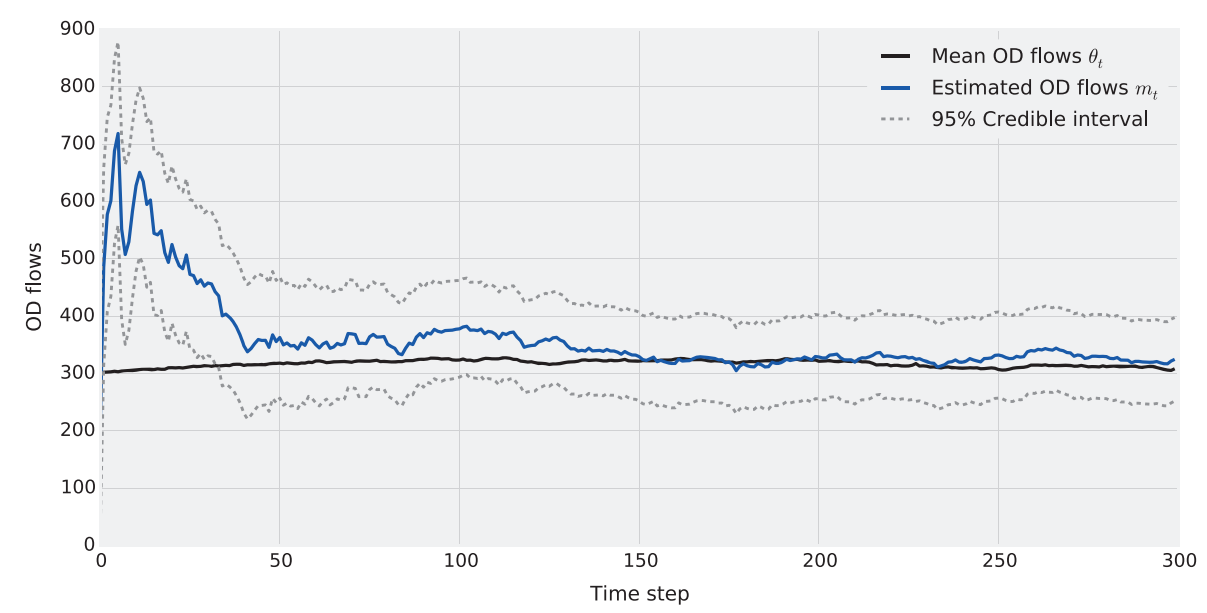

Figure 7. Origin-destination (OD) flows on OD pair $2 \rightarrow 13$. 
Table III. $M R A E_{T}$ for the Sioux Falls network with $R=30$.

\begin{tabular}{lcc}
\hline $\mathrm{T}$ & $M R A E_{T}$ & Standard deviation \\
\hline 0 & 0.9860 & 0.0000 \\
1 & 0.5898 & 0.0059 \\
10 & 0.5224 & 0.0104 \\
30 & 0.4237 & 0.0103 \\
100 & 0.2406 & 0.0070 \\
300 & 0.1018 & 0.0032 \\
\hline
\end{tabular}

The results are given in Table III, in which $M R A E_{T}$ at time $T=0$ is calculated for the initial estimate $\boldsymbol{m}_{0}$. With only one vector of observed link volumes, $M R A E_{T}$ decreases from the initial 0.9860 to 0.5898 . This result would approximately correspond to the application of a static model for which the prior OD matrix is far from the true one. As we gather more data, $M R A E_{T}$ is monotonically decreasing until it reaches 0.1018 for $T=300$. This indicates that the DLM is converging to the unobserved mean OD flows.

\section{DISCUSSION}

In this paper, we formulated a DLM for the estimation of time-varying day-to-day OD matrices. We have taken into consideration variability in OD flows, route flows, and link volumes. We applied Bayesian inference on mean OD flows given observed link volumes. We have also established the conditions upon which the mean OD flows may be estimated and tested the ideas in two networks from the literature. Our main hypothesis was that the DLM would converge to unobserved mean OD flows as more traffic volumes were observed over time, despite assuming uninformative prior distributions on mean OD flows. The proposed DLM converged to unobserved mean OD flows in both experiments carried out.

Some discussion is necessary on assumptions and limitations of our proposed model. We assumed that assignment matrices are known a priori. In practice, they have to be estimated in some way. Static models estimate assignment matrices by assuming that the transportation network is in equilibrium, and then resort to traffic assignment algorithms in order to obtain OD matrices, which are consistent with an equilibrium state. In this case, OD flows and assignment matrices are assumed constant over time. However, when we model OD flows as a dynamic system, the equilibrium assumption has been questioned [30]. When we model OD flows as a dynamic system, it is reasonable to assume that assignment matrices are also varying in time. There may be many sources of variability in assignment matrices, such as changes in congestion level due to varying OD flows as well as other factors, such as shifts in personal preferences, entrance of new users, and transitory changes in the transportation system. Then, assignment matrices should be estimated for each time period.

We envisage two alternatives to estimate assignment matrices: by direct sampling or by modeling. By direct sampling, we mean, for example, the use of plate scanning technology, which is becoming ubiquitous in large cities. Thereby, we can gather data on user's routes, enabling the estimation of route choice probabilities and then assignment matrices. In the second alternative, by modeling, we could embed route choice models in a DLM so as to jointly estimate mean OD flows and assignment matrices. These models could take into account the dynamic evolution of factors influencing route choices, such as the learning process of users and changes in infrastructure. Watling and Cantarella [31] have studied some learning models, which could be integrated in a DLM, and Hazelton and Parry [32] have recently explored by computational experiments the estimation of parameters of dynamic models of traveler behavior. We see the integration of such models as a promising research avenue, and we are already working on new models in which we jointly estimate mean OD flows and parameters of a route choice model.

Regarding covariance matrices, we are working on parsimonious specification of them. This is we do not have to estimate all elements of the covariance matrices. As an example of this approach, if we assume OD flows are independent and variances are proportional to mean OD flows in covariance matrix $\boldsymbol{\Sigma}_{t}^{x}$, we have to estimate only a proportionality constant, as shown by Hazelton [17]. A further 
possibility for the estimation of the covariance of OD flows is to use geographical data. Because of the spatial nature of OD flows, we may expect that OD flows, which share the same origins or destinations, are correlated.

The evolution covariance matrix $\mathbf{W}_{t}$ could be specified through a scalar discount factor $\varphi$, which represents the increase in uncertainty from time $t-1$ to $t$. Because the prior covariance matrix of OD flows at time $t$ is given by $\overline{\mathbf{C}}_{t}=\mathbf{C}_{t-1}+\mathbf{W}_{t}$, then we can write it as $\overline{\mathbf{C}}_{t}=(1 / \varphi) \mathbf{C}_{t-1}$, where $0<\varphi \leq 1$, which implies that $\mathbf{W}_{t}=((1-\varphi) / \varphi) \mathbf{C}_{t-1}$. Thus, we need to specify only the discount factor $\varphi$ and the starting covariance matrix $\mathbf{C}_{0}$, which synthesizes uncertainty on the prior OD matrix $\boldsymbol{m}_{0}$. When $\varphi=1.0$, we have $\mathbf{W}_{t}=0$ and then mean OD flows are constant over time. As $\varphi$ approaches zero, $\mathbf{W}_{t}$ increases, and variability in OD flows from time $t-1$ to $t$ is greater.

With respect to the covariance matrix $\boldsymbol{\Sigma}_{t}^{z}$ of observed volumes due to the errors in measurement, these may be estimated from the precision of the equipment used. If it is very precise, $\boldsymbol{\Sigma}_{t}^{z}$ could be set to very small values or even 0 .

Finally, our proposed DLM assumed a locally constant model for the dynamic evolution of mean OD flows. It could be extended to include trends and seasonal factors, which would also enable its application to forecasting link volumes.

\section{ACKNOWLEDGEMENTS}

The authors would like to thank Conselho Nacional de Desenvolvimento Científico e Tecnológico (CNPq) for the financial support.

\section{REFERENCES}

1. Ortúzar JD, Willumsen L. Modelling Transport (4th edn), Wiley: Chichester, 2011.

2. Cascetta E. Transportation Systems Analysis: Models and Applications (2nd edn), Springer: New York, NY, 2009.

3. Van Zuylen HJ, Willumsen LG. The most likely trip matrix estimated from traffic counts. Transportation Research Part B 1980; 281-293. DOI:10.1016/0191-2615(80)90008-9.

4. Willumsen LG. Simplified transport models based on traffic counts. Transportation 1981; 10: $257-258$. DOI:10.1007/BF00148462.

5. Cascetta E. Estimation of trip matrices from traffic counts and survey data: a generalized least squares estimator. Transportation Research Part B 1984; 16: 289-299. DOI:10.1016/0191-2615(84)90012-2.

6. Cascetta E, Nguyen S. A unified framework for estimating or updating origin/destination matrices from traffic counts. Transportation Research Part B 1988; 22B: 437-455. DOI:10.1016/0191-2615(88)90024-0.

7. Brenninger-Göthe $\mathrm{M}$, Jörnsten KO. Estimation of origin-destination matrices from traffic counts using multiobjective programming formulations. Transportation Research Part B 1989; 23B: 257-269. DOI:10.1016/ 0191-2615(89)90028-3.

8. Yang H, Sasaki T, Iida YAsakura Y. Estimation of origin-destination matrices from link counts on congested networks. Transportation Research Part B 1992; 26B: 417-434. DOI:10.1016/0191-2615(92)90008-K.

9. Yang H. Heuristic algorithms for the bilevel origin-destination matrix estimation problem. Transportation Research Part B 1995; 29B: 231-242. DOI:10.1016/0191-2615(95)00003-V.

10. Cascetta E, Postorino N. Fixed point approaches to the estimation of O/D matrices using traffic counts on congested networks. Transportation Science 2001; 35: 134-147. DOI:10.1287/trsc.35.2.134.10138.

11. Sherali HD, Narayanan ASivanandan R. Estimation of origin-destination trip-tables based on a partial set of traffic link volumes. Transportation Research Part B 2003; 37: 815-836. DOI:10.1016/S0191-2615(02)00073-5.

12. Shen W, Wynter L. A new one-level convex optimization approach for estimating origin-destination demand. Transportation Research Part B 2012; 46: 1535-1555. DOI:10.1016/j.trb.2012.07.005.

13. Caceres N, Romero LBenitez F. Inferring origin-destination trip matrices from aggregate volumes on groups of links: a case study using volumes inferred from mobile phone data. Journal of Advanced Transportation 2013; 47(7): 650-666. DOI:10.1002/atr.187.

14. Vardi Y. Network tomography: estimating source-destination traffic intensities from link data. Journal of the American Statistical Association 1996; 91: 365-377. DOI:10.2307/2291416.

15. Tebaldi C, West M. Bayesian inference on network traffic using link count data. Journal of the American Statistical Association 1998; 93: 557-573. DOI:10.2307/2670105.

16. Hazelton ML. Estimation of origin-destination matrices from link flows on uncongested networks. Transportation Research Part B 2000; 34: 549-566. DOI:10.1016/S0191-2615(99)00037-5.

17. Hazelton ML. Some comments on origin-destination matrix estimation. Transportation Research Part A 2003; 37: 811-822. DOI:10.1016/S0965-8564(03)00044-2.

18. Hazelton ML. Inference for origin-destination matrices: estimation, prediction and reconstruction. Transportation Research Part B 2001; 35: 667-676. DOI:10.1016/S0191-2615(00)00009-6. 
19. Manski C. Partial Identification of Probability Distributions, Springer Series in Statistics, Springer, 2003.

20. Hazelton ML. Network tomography for integer-valued traffic. Ann. Appl. Stat. 2015; 9(1): 474-506. DOI:10.1214/ 15-AOAS805.

21. Cremer M, Keller H. A new class of dynamic methods for the identification of origin-destination flows. Transportation Research Part B 1987; 21: 117-132. DOI:10.1016/0191-2615(87)90011-7.

22. Cascetta E, Inaudi DMarquis G. Dynamic estimators of origin-destination matrices using traffic counts. Transportation Science 1993; 27: 363-373. DOI:10.1287/trsc.27.4.363.

23. Ashok K, Ben-Akiva ME. Estimation and prediction of time-dependent origin-destination flows with a stochastic mapping to path flows and link flows. Transportation Science 2002; 36: 184-198. DOI:10.1287/trsc.36.2.184.563.

24. Lu Z, Rao W, Wu YJ, Guo LXia J. A kalman filter approach to dynamic od flow estimation for urban road networks using multi-sensor data. Journal of Advanced Transportation 2015; 49(2): 210-227. DOI:10.1002/atr.1292.

25. Singhal H, Michailidis G. Identifiability of flow distributions from link measurements with applications to computer networks. Inverse Problems 2007; 23(5): 1821.

26. Hazelton ML. Statistical inference for time varying origin-destination matrices. Transportation Research Part $B$ 2008; 42: 542-552. DOI:10.1016/j.trb.2007.11.003.

27. Parry K, Hazelton M. Bayesian inference for day-to-day dynamic traffic models. Transportation Research Part B 2013; 50: 104-115. DOI:10.1016/j.trb.2013.01.003.

28. Särkkä S. Bayesian Filtering and Smoothing, Cambridge University Press: New York, NY, USA, 2013.

29. West M, Harrison J. Bayesian Forecasting and Dynamic Models (2nd edn), Springer-Verlag: New York, NY, USA, 1997.

30. Lo HK, Watling DPCantarella GE. Editorial: day-to-day dynamics in transportation networks. Transportation Research Part B: Methodological 2016; 92(Part A): 1-2. DOI:10.1016/j.trb.2016.07.001.

31. Watling DP, Cantarella GE. Modelling sources of variation in transportation systems: theoretical foundations of day-to-day dynamic models. Transportmetrica B: Transport Dynamics 2013; 1(1): 3-32. DOI:10.1080/ 21680566.2013.785372.

32. Hazelton ML, Parry K. Statistical methods for comparison of day-to-day traffic models. Transportation Research Part B: Methodological in press2015. DOI:10.1016/j.trb.2015.08.005. 